\title{
Purely Organic Phosphor Sensitization Based Highly Efficient Electrofluorescence Material
}

\author{
Jiaxuan Wang ${ }^{1}$, Baoyan Liang ${ }^{2}$, Jinbei $\mathrm{Wei}^{1}$, Zhiqiang $\mathrm{Li}^{2}$, Yincai Xu${ }^{1}$, Tong Yang ${ }^{1}$, \\ Chenglong $\mathrm{Li}^{1 *}$, Yue Wang ${ }^{1,2 *}$
}

${ }^{1}$ State Key Laboratory of Supramolecular Structure and Materials, College of Chemistry, Jilin University, Qianjin Avenue, Changchun, 130012, P. R. China

${ }^{2}$ Jihua Laboratory, 28 Huandao South Road, Foshan, 528200, Guangdong Province, P. R. China.

* Authors to whom correspondence should be addressed:

chenglongli@jlu.edu.cn (CL); yuewang@jlu.edu.cn (YW) 
Abstract: Pure organic room temperature phosphorescence (RTP) materials are considered as potential candidates for replacing precious metal-based complexes to fabricate highly efficient organic light emitting devices (OLEDs). However, for reported RTP materials, low photoluminescence quantum yields (PLQYs) in thin film state seriously impede their applications in OLEDs. On the other hand, how using normal organic fluorescence materials to fabricate OLEDs with an internal quantum efficiency (IQE) over $25 \%$ remains a great unaddressed issue beyond the thermally activated delayed fluorescence (TADF) sensitization approach. Here, we establish a strategy to construct highly efficient OLEDs based on pure organic RTP material sensitized fluorescence emitter. The key point for our strategy is that benzimidazole-triazine molecules (PIM-TRZ), 2,6-di(phenothiazinyl)naphthalene ( $\beta$-DPTZN) and 5,6,11,12-tetraphenylnaphthacene (rubrene) were screened as host, phosphor sensitizer and fluorescent emitter, respectively. Detail photophysical characterizations demonstrate that the host material PIMTRZ with unique RTP nature is critical for achieving phosphor sensitizing process. As an organic RTP compound, the singlet and triplet state energy levels of $\beta$-DPTZN perfectly match with those of PIMTRZ, resulting in the formation and lasting existence of phosphor's excitons in emitting layer. The large overlap between the absorption spectrum of rubrene and PL spectrum of PIM-TRZ:10\% $\beta$-DPTZN film can facilitate the Förster energy transfer from the triplet $\beta$-DPTZN to the singlet rubrene and the finally displayed fluorescence is derived from singlet excited states of rubrene. The perfect collocation of host, phosphorescent sensitizer and fluorescent emitter in the emitting layer promise the predominant performance of the devices with external quantum efficiency (EQE) of 15.7\%. The PLQY of emitting layer is $60.3 \%$, and therefore about $90 \%$ carrier injection induced excitons are harvested for light emission. We present a new strategy to fabricate efficient fluorescent devices by employing ingenious combination of host, phosphorescent sensitizer and fluorescent emitter, which is significant to the development of OLEDs. 
Over the past three decades, material scientists continuously devote to the development of organic emitters that can harvest both singlet and triplet excited states generated by electrical injection. In organic lighting emitting devices (OLEDs) ${ }^{1}$, the recombination between hole and electron carriers results in the formation of singlet and triplet excited states with a ratio of 1:3 based on spin statistic principle. Therefore, for OLEDs with traditional fluorescent materials as emitters, the maximum internal quantum efficiency (IQE) is limited to $25 \%$. In this context, phosphorescent complexes containing precious heavy metal, which exhibit heavy-atom induced strong spin-orbit coupling effect and enhanced intersystem crossing, were first utilized to fabricate OLEDs with IQE of $100 \%{ }^{2}$. Sequentially, metal free thermally activated delayed fluorescence (TADF) materials were successfully employed emitters to construct OLEDs with unit IQE, which was realized by transforming triplet excited states into singlet excited states through reverse intersystem crossing (RISC) process ${ }^{3}$. The organic radical-based OLEDs with doublet emission feature intelligently avoided the limitation of spin statistic rule and achieved very high IQE ${ }^{4}$. The proposal of hybridized local and charge-transfer (HLCT) excited state developed a unique pass way to harvest singlet and triplet excitons in OLEDs ${ }^{5}$.

Except for the above-mentioned methods of improving the IQE of OLEDs, it is well known that purely room-temperature phosphorescence (RTP) organic materials-based OLEDs also have the potential to approach 100\% IQE. Recently, a large number of RTP organic molecules have been reported $^{6-13}$. However, only one example displayed higher electroluminescence (EL) efficiency reported by our group so far $^{14}$. During the investigation of pure organic phosphorescent OLEDs, we confront the insuperable predicament: low photoluminescent (PL) efficiency or even disappear of RTP in thin film state. Obviously, fabricating high-performance OLEDs with RTP materials as emitters directly is not a desired and even closed avenue. Therefore, to pursue high efficiency RTP organic material based OLEDs remains a great challenge, and new strategies for constructing RTP based high efficiency pure organic electroluminescence materials should be focused; urgently. In this contribution, RTP materials were employed as sensitizer and fluorescent materials as emitter to fabricate high-performance OLEDs. 

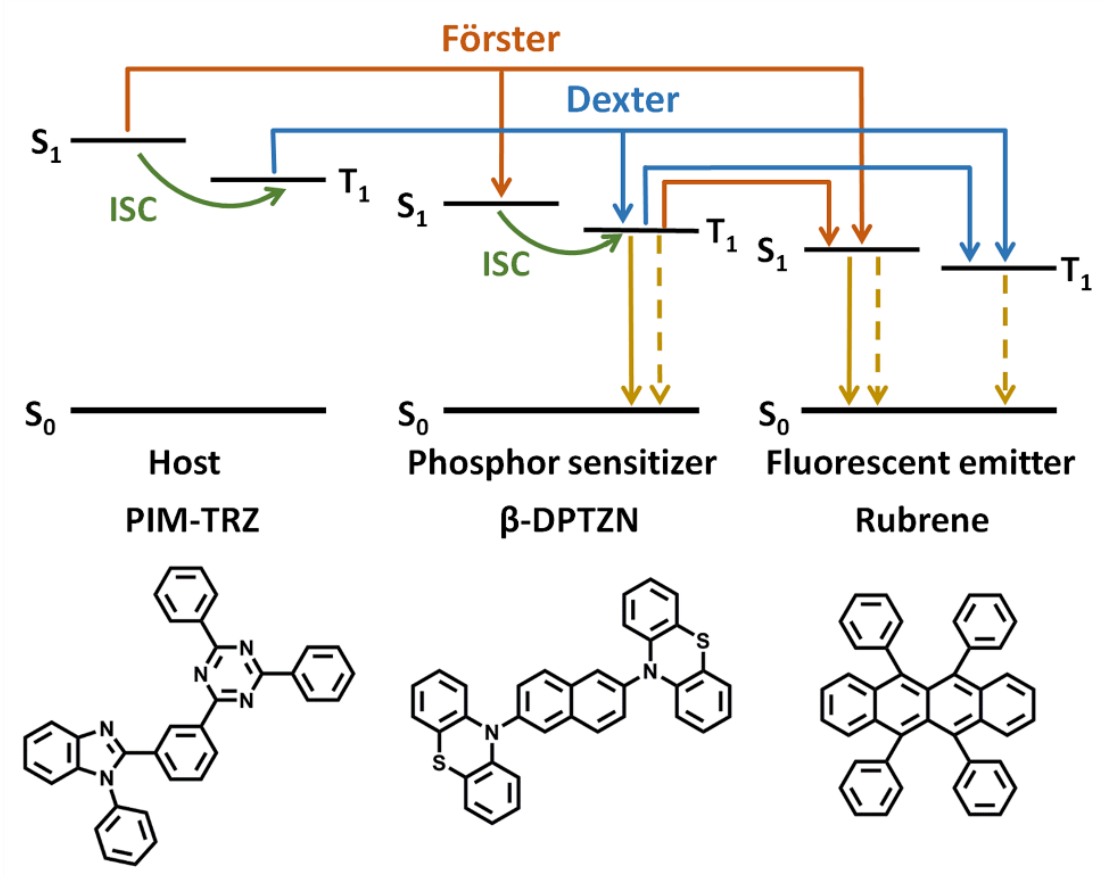

Scheme 1. Phosphor sensitizing mechanism and materials. Schematic illustration of energy transfer process and molecular structures of $\beta$-DPTZN, PIM-TRZ and rubrene.

To construct highly efficient OLEDs, a unique strategy of RTP molecule sensitizing fluorescent emitter was proposed and verified. Based on a large number of experimental attempts, $\beta$-DPTZN ${ }^{14}$ (Scheme 1) was employed as the phosphor sensitizer, PIM-TRZ ${ }^{15}$ was optimized as the host, and 5,6,11,12-tetraphenylnaphthacene (rubrene) with a high photoluminescence quantum yields (PLQYs) was screened as the fluorescent emitter. To evaluate EL performance of phosphor sensitized fluorescence, the rubrene-doped OLEDs were fabricated and the adopted devices structure was [ITO/NPB (30 nm)/TCTA (5 nm)/ PIM-TRZ:10\% $\beta$-DPTZN:X\% Rubrene (20nm)/TPBI (50 nm)/LiF $(1 \mathrm{~nm}) / \mathrm{Al}(100 \mathrm{~nm})]$ (device $1, \mathrm{X}=0 \mathrm{wt}$; device $2, \mathrm{X}=0.3 \mathrm{wt}$; device $3, \mathrm{X}=0.5 \mathrm{wt}$; device $4, \mathrm{X}=0.8$ $w t$ and device $5, X=1.0 \mathrm{wt}$ ). For device 1 without rubrene dopant, $\beta$-DPTZN acts as emitter directly ${ }^{14}$. Fig. 1a shows the schematic diagrams of the OLEDs and energy levels of the materials used in devices. The molecular structures of NPB, TCTA, TPBI are presented in Fig. 1b. The EL spectra of devices 1-5 are shown in Fig. 1c. The EL performances are summarized in Fig. 1d-f and Table 1. For comparison, device 6 of [ITO/NPB (25 nm)/TCTA (10 nm)/PIM-TRZ:0.5\% Rubrene (25 nm)/TPBI (45 nm)/LiF (1 $\mathrm{nm}) / \mathrm{Al}(100 \mathrm{~nm})]$ without phosphor sensitizer was fabricated. The maximum external quantum efficiencies (EQEs) of the devices 2-5 at different rubrene doping concentrations were 15.7\%, 15.3\%, $15.4 \%$ and $14.5 \%$, respectively, while the EQEs at $1000 \mathrm{~cd} / \mathrm{m}^{2}$ were $11.9 \%, 11.9 \%, 12.5 \%$ and $11.7 \%$. Through a comprehensive analysis to the parameters of devices 2-5, device 2 with rubrene dopant 
concentration of $0.3 \%$ achieved the maximum EQE of $15.7 \%$, luminance of $24260 \mathrm{~cd} / \mathrm{m}^{2}$, power efficiency (PE) of $53.2 \mathrm{~lm} / \mathrm{W}$, current efficiency (CE) of $47.6 \mathrm{~cd} / \mathrm{A}$.
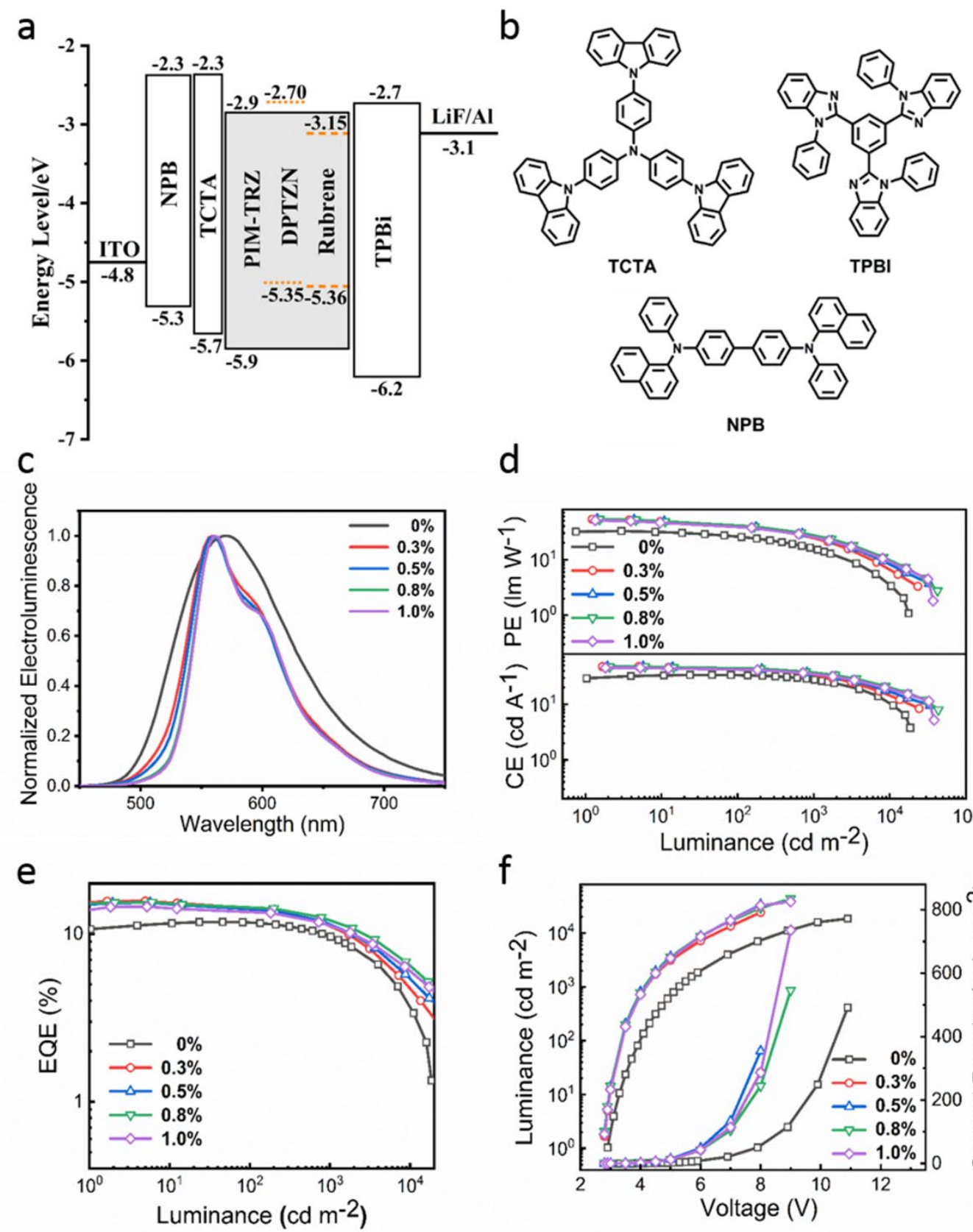

d
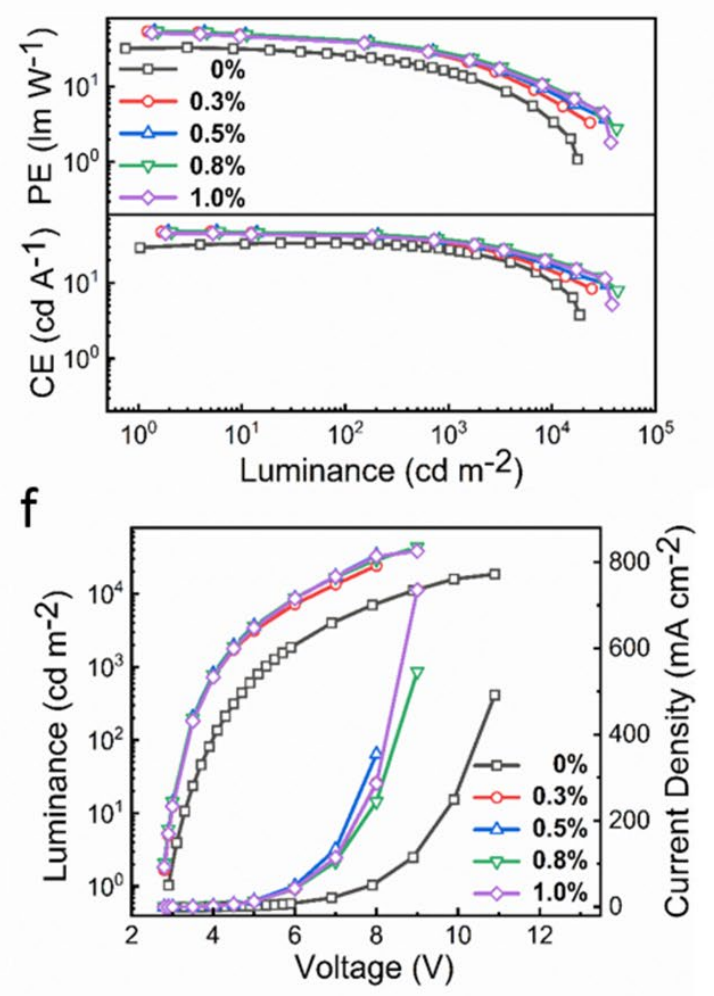

Fig. 1. Device structure and EL performance of Phosphor sensitized OLEDs. (a) Schematic diagram of the OLEDs and energy levels of the compounds used in fluorescent OLEDs. (b) Molecular structures of NPB, TCTA and TPBI used in devices. (c) EL spectra of devices $1-5$ at $100 \mathrm{~cd} / \mathrm{m}^{2}$. (d) PE-Brightness-CE characteristics of devices 1-5. (e) EQE-Brightness and (f) Current density-Voltage-Brightness (J-V-L) characteristics of devices 1-5. ( $X$ represent the doping concentration of rubrene. device $1, X=0 \%$; device $2, X=0.3 \%$; device $3, X=0.5 \%$; device $4, X=0.8 \%$; device $5, X=1.0 \%$ ).

Devices 2-5 presented not only high efficiencies but also low efficiency roll-offs. The 
photoluminescence quantum yields (PLQYs) of the doped films with rubrene dopant concentrations of 0.3-1.0 wt \% are 60.3-55.0\% (Table S1) and the experimentally recorded EQE values of the devices are 15.6-14.5\%. The maximum theoretical EQE values of devices $2-5$ are calculated to be $18.1-16.5 \%$ based on the PLQYs and light out-coupling efficiency $\left(\eta_{\text {out }}\right)$ of $30 \%$. The achieved EQE of devices 2-5 approached the maximum theoretical EQEs. Therefore, most of the charge injection-induced triplet excited states in the devices were harvested. For device 1 without rubrene, the EQE of $11.5 \%$ was recorded suggesting that fluorescent dopant can obviously enhance the EL efficiency. Device 6 without phosphor sensitizer displayed the EQE of 3.8\% and its EL spectrum exhibited an additional emission shoulder at around $500 \mathrm{~nm}$ except for the fluorescence of rubrene, which should be attributed to the interface exciplex emission formed between TCTA and PIM-TRZ (Fig. S1). In the absence of phosphor sensitizer, device displayed a poor performance. We also fabricated a series devices with different doping concentrations of $\beta$-DPTZN, the device structures are [ITO/NPB (30 nm)/TCTA (5 nm)/PIMTRZ:Y\% $\beta$-DPTZN:0.5\% Rubrene (20nm)/TPBI (50 nm)/LiF (1 nm)/Al (100 nm)] (device 7, Y= 3 wt; device $8, Y=5 \mathrm{wt}$; device $9, \mathrm{Y}=10 \mathrm{wt}$ and device $10, \mathrm{Y}=15 \mathrm{wt}$ ). The best performance of OLEDs can be accessed when the doping concentration of $\beta$-DPTZN is $10 \%$. The performance of devices is shown in Fig. S2 and Table S2. As increasing the doping concentration of $\beta$-DPTZN, the cascade energy sequential transfer from PIM-TRZ to DPTZN to rubrene was facilitated. However, the Dexter energy transfer from triplet of $\beta$-DPTZN to triplet of rubrene should be increased as well, which can lead to the decline of EL efficiency at high doping concentration of $\beta$-DPTZN. Although some heavy metal complex based phosphor sensitized fluorescence OLEDs have been reported ${ }^{16-23}$, here we demonstrated purely organic phosphor sensitized fluorescence OLEDs with higher efficiency for the first time.

Table 1. Electroluminescent parameters of the devices 1-6.

\begin{tabular}{ccccccc}
\hline Device & $\begin{array}{c}\mathrm{V}_{\text {on }} \\
{[\mathrm{V}]^{[\mathrm{a}]}}\end{array}$ & $\begin{array}{c}\mathrm{L}_{\max } \\
{\left[\mathrm{cd} \mathrm{m}^{-2}\right]^{[\mathrm{b}]}}\end{array}$ & $\begin{array}{c}\mathrm{CE} \\
{\left[\mathrm{cd} \mathrm{A}^{-1}\right]^{[\mathrm{c}]}}\end{array}$ & $\begin{array}{c}\mathrm{PE} \\
\left.[\mathrm{lm} \mathrm{W}]^{-1}\right]^{[\mathrm{c}]}\end{array}$ & $\begin{array}{c}\mathrm{EQE} \\
{[\%]^{[\mathrm{c}]}}\end{array}$ & $\begin{array}{c}\mathrm{CIE} \\
(x, y)^{[\mathrm{d}]}\end{array}$ \\
\hline Device 1 14 & 2.9 & 18500 & $33.8,33.3,27.7$ & $32.6,25.5,16.4$ & $11.5,11.6,9.6$ & $0.46,0.52$ \\
Device 2 & 2.8 & 24260 & $47.6,42.4,36.1$ & $53.2,38.1,28.4$ & $15.7,14.0,11.9$ & $0.46,0.52$ \\
Device 3 & 2.8 & 33820 & $47.8,42.9,37.0$ & $53.6,38.5,29.1$ & $15.3,13.8,11.9$ & $0.47,0.52$ \\
Device 4 & 2.8 & 43360 & $47.8,43.9,38.8$ & $53.4,39.4,30.5$ & $15.4,14.2,12.5$ & $0.48,0.51$ \\
Device 5 & 2.8 & 38210 & $45.5,41.7,36.8$ & $50.8,37.4,28.9$ & $14.5,13.3,11.7$ & $0.48,0.51$ \\
Device 6 & 3.0 & 10900 & $12.1,12.0,10.8$ & $10.5,9.5,6.2$ & $3.8,3.7,3.4$ & $0.47,0.51$
\end{tabular}

[a] turn-on votage at $1.0 \mathrm{~cd} \mathrm{~m}^{-2} .{ }^{[b]}$ maximum luminance. ${ }^{[\mathrm{c}]}$ in the order of maximum, then the values recorded 
at 100 and $1000 \mathrm{~cd} \mathrm{~m}^{-2}$. [d] recorded at $100 \mathrm{~cd} \mathrm{~m}^{-2}$.

To understand the mechanism of organic phosphor sensitizer induced enhancement of EL efficiency, a series of detail characterizations were carried. Fig. S3a shows the absorption spectrum of rubrene in toluene solution and the PL spectra of [PIM-TRZ:10 wt\% $\beta$-DPTZN] blending film and rubrene in toluene solution. The absorption spectrum of rubrene in toluene solution and the PL spectrum of PIM-TRZ:10 wt\% $\beta$-DPTZN blending film have a large overlap, which suggests the facile Förster energy transfer channel from phosphor sensitizer to rubrene. The spectral overlap integral $J(\lambda)$ of PL spectrum of [PIM-TRZ:10 wt\% $\beta$-DPTZN] blending film and extinction spectrum of rubrene could be obtained. The Förster radii is estimated to be $29.64 \mathrm{~nm}$, indicating the long-range Förster energy transfer is efficient at a low doping concentration of rubrene (for the detail information see Supporting Information $)^{23,24}$. Fig. S3b shows the PL spectra of [PIM-TRZ:10 wt\% $\beta$-DPTZN], [PIM-TRZ:0.5 wt\% rubrene] and [PIM-TRZ:10 wt\% $\beta$-DPTZN:0.5 wt\% rubrene] blending films. In the PL spectra of [PIMTRZ:10 wt \% $\beta$-DPTZN:0.5 wt\% rubrene] blending film, the emission of PIM-TRZ from 350 to $500 \mathrm{~nm}$ almost disappeared compared with that of [PIM-TRZ:0.5 wt\% rubrene] blending film. The energy transfer from PIM-TRZ to rubrene is deficient in the film without $\beta$-DPTZN. This demonstrated the existence of phosphor sensitizer $\beta$-DPTZN is indispensable, it could sufficiently promote the energy transferred from PIM-TRZ to rubrene. Therefore, $\beta$-DPTZN is capable of being the bridge between host and fluorescent emitter by setting up an efficient energy transfer passageway. Furthermore, the PL spectra of films became narrow after doping rubrene indicating the energy transfer from $\beta$-DPTZN to rubrene. 

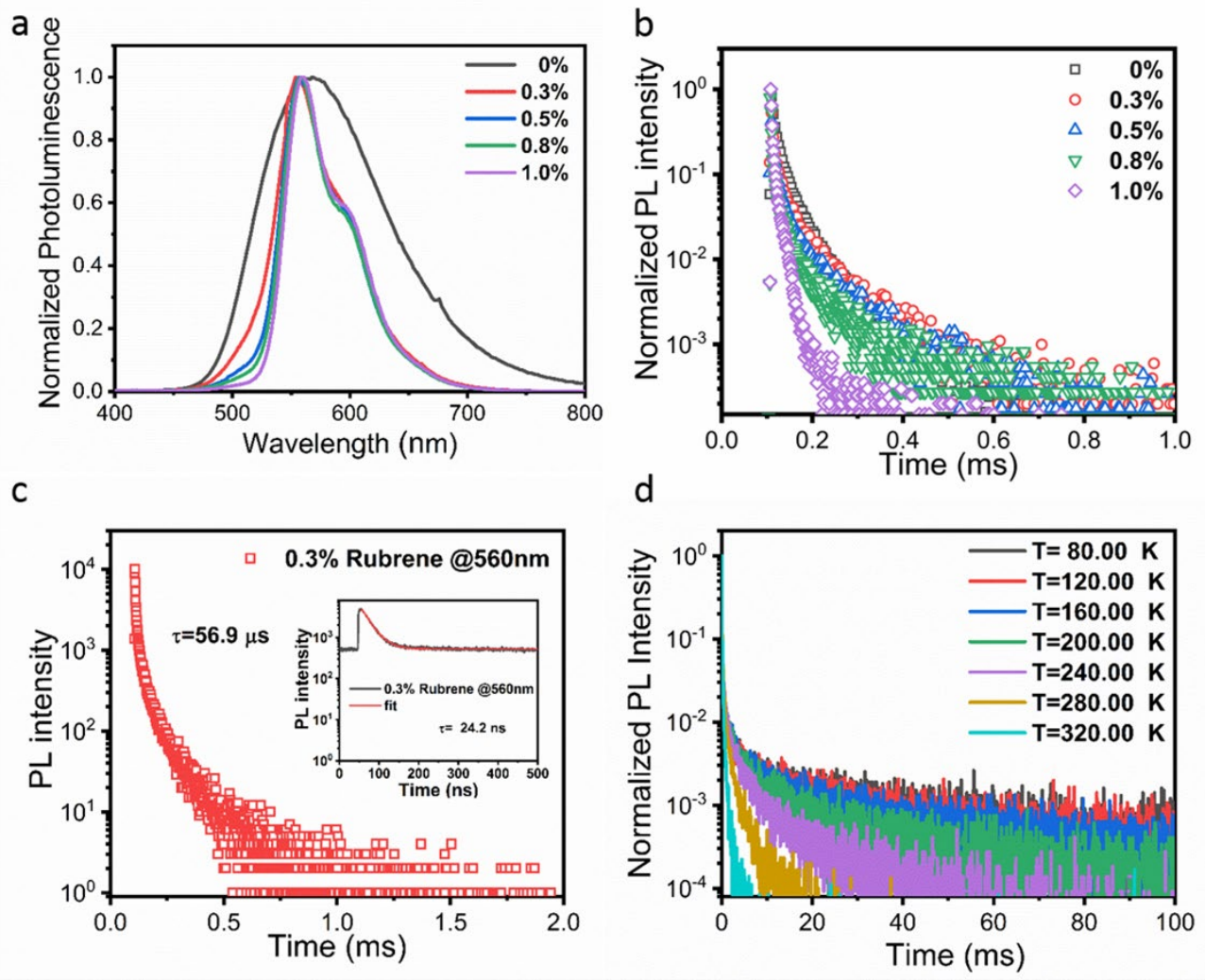

Fig. 2. Photophysic property of phosphor sensitized films. (a) Room temperature PL spectra of [PIM-TRZ:10 $w t \% \beta$-DPTZN:X wt\% rubrene blending] film, $X=0,0.3,0.5,0.8$ and 1.0. (b) Room temperature transient PL spectra of [PIM-TRZ:10 wt $\% \beta$-DPTZN:X wt \% rubrene] blending film, $X=0,0.3,0.5,0.8$ and 1.0. (c) Transient PL spectra of [PIM-TRZ:10 wt\% $\beta$-DPTZN:0.3 wt\% rubrene] blending film. (d) Temperature-dependent transient PL spectra of [PIM-TRZ:10 wt\% $\beta$-DPTZN:0.3 wt\% rubrene] blending film.

The steady and transient PL spectra of films with different rubrene doping concentrations are shown in Fig. 2a and 2b. The transient PL spectrum (Fig. 2c) of [PIM-TRZ:10 wt $\% \beta$-DPTZN:0.3 wt $\%$ rubrene] blending film exhibited decay lifetime of $56.9 \mu$ s at $560 \mathrm{~nm}$, which is shorter than exciton decay lifetime $(87.0 \mu \mathrm{s})$ of film without rubrene. Table S1 presents the decay lifetimes for the films with different doping concentration of rubrene. The exciton decay lifetimes gradually decrease upon increasing the doping concentration, indicating the energy transfer from phosphor to the fluorescent emitter. The temperature-dependent transient PL spectra (Fig. 2d) show that upon improving temperature the decay lifetimes become shorter and shorter. Therefore, the blending film of [PIMTRZ:10 wt $\% \beta$-DPTZN:0.3 $\mathrm{wt} \%$ rubrene] is dynamically phosphorescent characteristic, which is attributed to the photophysical nature of $\beta$-DPTZN. The temperature-dependent transient PL spectra of films with different rubrene doping concentrations exhibited identical behavior (Fig. S4). Additionally, for the [PIM-TRZ:10 wt\% $\beta$-DPTZN:0.3 wt\% rubrene] blending film, the time-dependent PL spectra 
shown gradual decrease of intensity with increase of delayed time (Fig. S5). The PL spectrum recorded at $120 \mathrm{~ns}$ displayed a slight shoulder at around $500 \mathrm{~nm}$, which was ascribed to the residual emission of $\beta$-DPTZN. Beyond $120 \mathrm{~ns}$, there is negligible residue of $\beta$-DPTZN emission in the spectra, further confirming the energy transfer process from host PIM-TRZ to phosphor sensitizer $\beta$-DPTZN, subsequently to fluorescent emitter rubrene. The steady PL spectra of [PIM-TRZ:10 wt $\% \beta-$ DPTZN:0.3-1.0 wt\% rubrene] films represented the emission feature of rubrene.
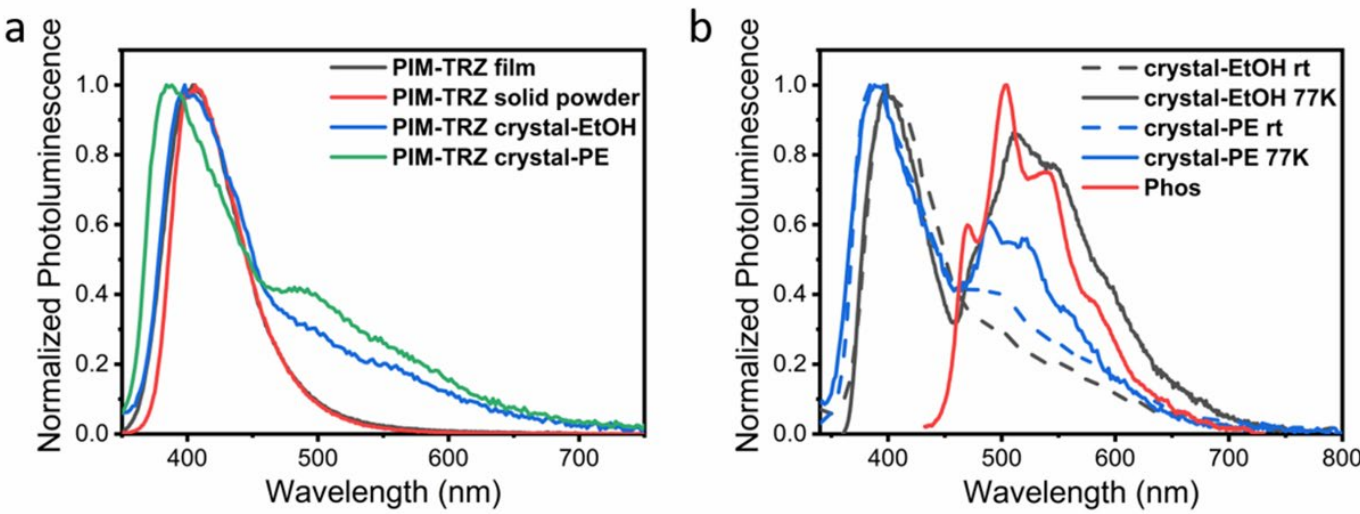

Fig. 3. RTP nature of host material PIM-TRZ. (a) Room temperature PL spectra of PIM-TRZ in different states (solid powder, film, crystal-EtOH and crystal-PE). (b) PL spectra of two forms of crystal at room temperature and $77 \mathrm{~K}$ and phosphorescence (Phos) spectra of PIM-TRZ in toluene at $77 \mathrm{~K}$.

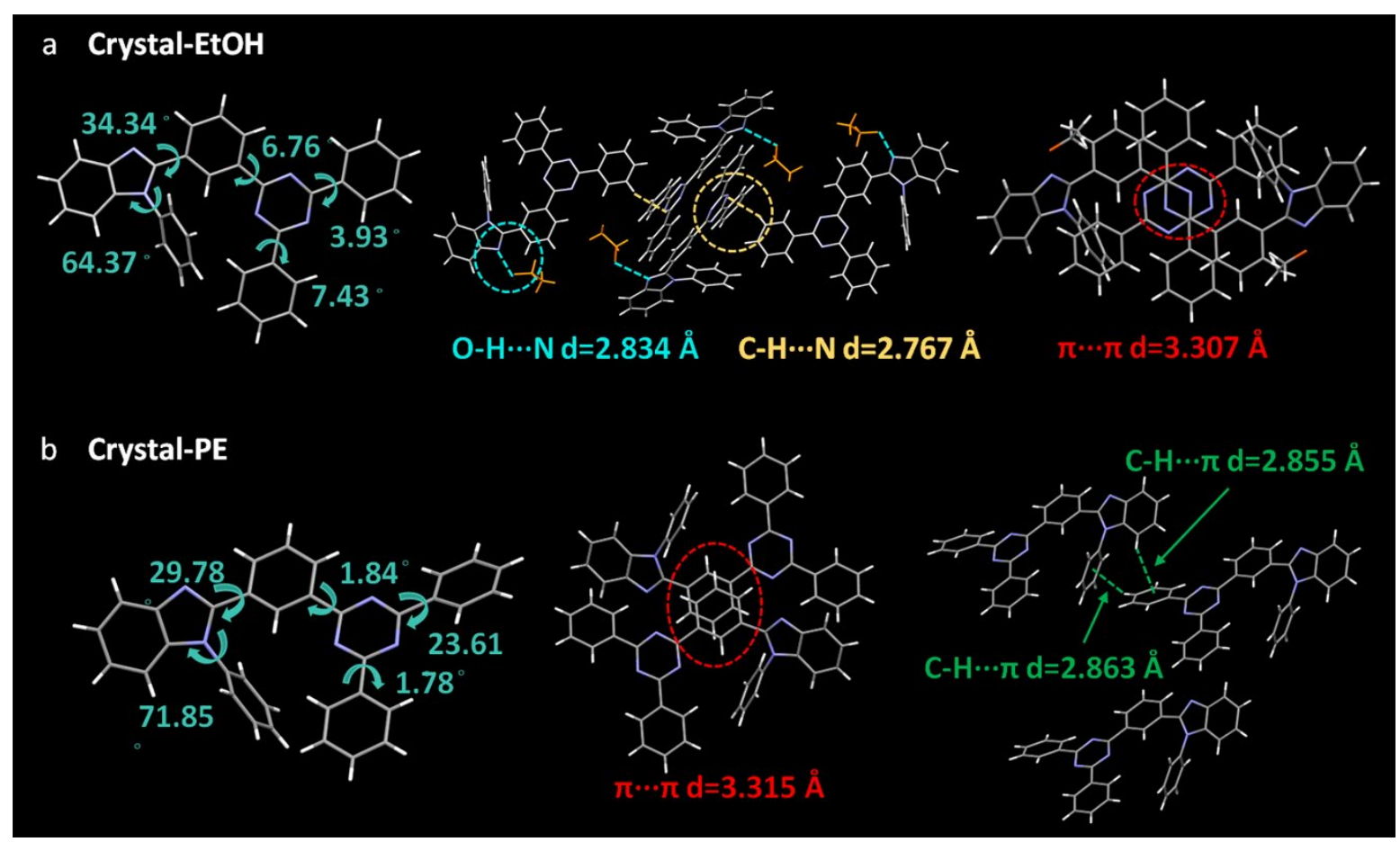

Fig. 4. Molecular conformations and packing modes of PIM-TRZ in crystalline phases. (a) Molecular structure of PIM-TRZ in crystal-EtOH from single-crystal XRD. Intermolecular interactions and packing modes in crystal-EtOH. (b) Molecular structure of PIM-TRZ in crystal-PE from single-crystal XRD. Intermolecular interactions and packing modes in crystal-PE. 
The host material PIM-TRZ played a critical role for the phosphor sensitizing fluorescent emitter. Some other host materials, which were often used in OLEDs, haven't displayed the similar performance. To figure out the mechanism of PIM-TRZ enhanced phosphor sensibility, the detail phtophysical characterizations were put into effect. Fig. 3a presents the PL spectra of PIM-TRZ in different states. The film was prepared by vacuum sublimation and deposition, while solid powder sample was directly collected from sublimation tube. The crystal-PE was prepared by slowly evaporating a mixed solution of $\mathrm{CH}_{2} \mathrm{Cl}_{2}$ and petroleum ether (PE), while the crystal-EtOH was generated by delay evaporation of a mixed solution of $\mathrm{CH}_{2} \mathrm{Cl}_{2}$ and ethyl alcohol $(\mathrm{EtOH})$. The film and solid powder samples only exhibit a sharp emission peak at around $400 \mathrm{~nm}$. For the two crystalline samples, the emission maximum at around $400 \mathrm{~nm}$ was accompanied with remarkable shoulders at 500 and $550 \mathrm{~nm}$. Furthermore, crystalPE displays more intensity shoulder emission compared with crystal-EtOH. For the four samples, the emission maxima at around $400 \mathrm{~nm}$ only exhibit a short life time of several nanoseconds (Fig. S6). The crystalline samples show long decay lifetimes (1.15 ms for crystal-PE and $9.9 \mathrm{~ms}$ for crystal-EtOH) at $560 \mathrm{~nm}$ of shoulder emission. Although the neat PIM-TRZ solid thin-film and solid powder show very weak emission at around $560 \mathrm{~nm}$, long life-times excited states also were detected (8.0 $\mu$ s for film and $2.1 \mu$ s for solid powder). The phosphorescence spectra of PIM-TRZ in toluene solution at $77 \mathrm{~K}$ and the shoulder emission of two crystalline samples display similar profiles at around $550 \mathrm{~nm}$ (Fig. 3b). Therefore, the shoulder emission is attributed to the phosphorescence of PIM-TRZ in different crystalline phases. The temperature-dependent transient PL spectra (Fig. S7) of two crystals reveal that the decay lifetimes are reduced upon the temperature increasing, which substantiates that PIM-TRZ possess the RTP nature. The temperature-dependent transient PL spectra detected at $560 \mathrm{~nm}$ are shown in Fig. S8, which also confirm the RTP feature of PIM-TRZ film and solid powder. The unique RTP characteristic of PIM-TRZ should be responsible for the special phosphor sensitizing behavior in [PIMTRZ/ $\beta$-DPTZN/rubrene] composite film system. The PIM-TRZ matrix with RTP nature may provide a friendly environment that is beneficial to spin-orbital coupling (SOC) effect and intersystem crossing process $^{25}$. The PL spectra of PIM-TRZ, $\beta$-DPTZN and rubrene in toluene solution at $77 \mathrm{~K}$ and corresponding phosphorescence spectra are depicted in Fig. S9. The $E_{\mathrm{S} 1}$ of PIM-TRZ and $\beta$-DPTZN are $3.52 \mathrm{eV}$ and $3.23 \mathrm{eV}$, respectively, estimated from the PL spectra at $77 \mathrm{~K}$. The $E_{\mathrm{T} 1}$ of PIM-TRZ and $\beta$ DPTZN are $2.76 \mathrm{eV}$ and $2.64 \mathrm{eV}$, respectively, appraised from the phosphorescence spectra. The $E_{\mathrm{S} 1}$ of rubrene is $2.29 \mathrm{eV}$, calculated from the PL spectrum at $77 \mathrm{~K}$. The $E_{\mathrm{T} 1}$ of PIM-TRZ is higher than $E_{\mathrm{T} 1}$ of 
$\beta$-DPTZN, and the $E_{\mathrm{S} 1}$ of rubrene is lowest. The well-matched triplet and singlet energy levels are beneficial to the cascade energy transfer from PIM-TRZ to $\beta$-DPTZN to rubrene, ensuring the efficient fluorescence emission.

To probe into the cause of phase dependent phosphorescent property of PIM-TRZ, the crystal structures of crystal-EtOH and crystal-PE were studied (Fig. 4 and Fig. S10). PIM-TRZ molecules adopted obvious different conformations and packing modes. The dihedral angles between 1,3,5triazine and three attached benzene rings are depicted in Fig. 4a and 4b, respectively, revealing the diversity of molecular conformations. Furthermore, in two crystals different intermolecular $\pi \cdots \pi$ interaction states were presented. In crystal-PE intermolecular $\pi \cdots \pi$ interactions take place between the phenyl linker connecting 1,3,5-triazine and benzoimidazole, while in crystal-EtOH that exist between two adjacent 1,3,5-triazine rings. In the crystals, there are abundant intermolecular $\mathrm{C}-\mathrm{H} \cdots \pi$ interactions. The strong intermolecular interaction can enhance the rigidity of PIM-TRZ molecules and restrict triplet nonradiative transition, as further is beneficial to the radiative transition of triplet excitons. The theoretical calculations depict the SOC constants of PIM-TRZ of different crystal packing modes in Table S1. The SOC matrix elements (SOCME) study reveals the possibility of intersystem crossing and phosphorescent emission. The SOCME between $\mathrm{T}_{1}$ and $\mathrm{S}_{0}$ of crystal-EtOH and crystal-PE are 0.703 and $0.785 \mathrm{~cm}^{-1}$, respectively. SOCME between $\mathrm{S}_{1}$ and $\mathrm{T}_{1}$ of crystal-EtOH and crystal-PE are 0.371 and $0.790 \mathrm{~cm}^{-1}$, respectively. The SOC values demonstrated that the two crystals indeed have the potential to display RTP behavior. The SOC calculation results also identified that the RTP property of PIM-TRZ based samples is molecular conformation and packing dependent, therefore, PIM-TRZ exhibits phase dependent RTP property.

The schematic illustration of energy transfer processes is presented in Scheme 1. The carrier injections generate singlet and triplet exited PIM-TRZ molecules and then energy transfer from PIMTRZ to $\beta$-DPTZN via Förster and Dexter processes. Singlet excited $\beta$-DPTZN can change into triplet excited $\beta$-DPTZN by intersystem crossing (ISC) process. Finally, the triplet exited energy of $\beta$-DPTZN transfer to rubrene molecules by Förster process, resulting in large amount of singlet exited rubrene molecules. The RTP property of host material PIM-TRZ can promote ISC process and suppress the nonradiative triplet energy consumption and therefore, ensure the Dexter triplet energy transfer from PIM-TRZ to $\beta$-DPTZN. On the other hand, the RTP nature of PIM-TRZ can also stabilize the triplet exited $\beta$-DPTZN molecules and guarantee Förster energy transfer from triplet $\beta$-DPTZN to singlet 
rubrene, which is the most important process for achieving high EL efficiency. The dominant energy transfer form from $\beta$-DPTZN to rubrene is the Förster energy transfer process rather than Dexter energy transfer, because of the spatial separation of $\beta$-DPTZN and rubrene with the low doping concentration of rubrene.

We have successfully constructed a class of electroluminescence films based on purely organic phosphor sensitizing fluorescence emitter and originally demonstrated a unique approach to achieving highly efficient OLEDs. The strategy reported in this study founded a new potential channel to realize electroluminescence with IQE of $100 \%$ beyond the reported precious heavy metal-based phosphorescence, TADF, radical emitter and HLCT emission OLEDs. The experimental results verified that RTP sensitized fluorescent materials can break the limitation of $25 \%$ IQE for normal electrofluorescence devices. Significantly, the RTP sensitizing fluorescence emitters are noble metal free and should be the most promising and low-cost candidates for harvest both singlet and triplet excitons generated by carrier injection in OLEDs. We fabricated an efficient fluorescent device with a maximum EQE of $15.7 \%$ and low efficiency roll-off by using the pure organic compounds with phosphorescent characteristic as host and sensitizer. We identified that PIM-TRZ host with RTP feature is a key factor for achieving highly efficient Förster energy transfer from phosphor to fluorescent molecules. The developed method in this contribution can avoid the disadvantage of RTP materials with low PLQYs, which restrict their application in electroluminescence, and establish a new avenue to construct high-performance OLEDs based on normal and classic fluorescence emitters.

\section{Acknowledgements}

CL and WY are grateful for financial support from the National Natural Science Foundation of China (Grant No. 21935005), the National Key R\&D Programme of China (Grant No. 2020YFA0714601) and Program for JLU Science and Technology Innovative Research Team (Grant No. 2019TD-33).

\section{Author Contributions}

JW synthesized and characterized the phosphor sensitizing materials and performed device fabrications and characterizations. BL and JW synthesized host material. YX and TY optimized the devices. ZL performed the electronic structure calculations. YW proposed and designed the phosphor sensitizing material system. JW and YW wrote the manuscript. CL and YW conceived the project, 
supervised the work.

\section{Additional information}

Supplementary information accompanies this paper at [to be completed in proofs]

\section{Competing financial interests}

The authors declare no competing interests. 


\section{Methods}

Materials purification. All materials used in this study were purified by sequentially crystallization, column chromatography and two times vacuum sublimation.

Photoluminescence measurements. The UV-Vis absorption spectra recorded by a Shimadzu UV-2550 spectrophotometer. The steady PL spectra and PLQYs were using an Edinburgh FLSP920 fluorescence spectrophotometer equipped with a xenon arc lamp (Xe750) and integrating sphere. Room temperature, temperature dependent and time dependent transient spectra were recorded using FLS920 fluorescence lifetime measurement system with $365 \mathrm{~nm}$ LED excitation source and a microsecond flash-lamp (uF750).

Device fabrication and characterizations. Glass substrates pre-coated with indium tin oxide (ITO) with a sheet resistance of $15 \Omega$ per square were thoroughly cleaned in ultrasonic bath of tetrahydrofuran, detergent, deionized water, acetone and isopropyl alcohol and treated with plasma for 5 min in sequence. Organic layers were deposited onto the ITO-coated glass substrates by thermal evaporation under high vacuum $\left(<5 \times 10^{-4} \mathrm{~Pa}\right)$. Cathode was patterned using a shadow mask with an array of $2.0 \mathrm{~mm} \times 2.5 \mathrm{~mm}$ openings. Deposition rates are $1 \AA \mathrm{s}^{-1}$ for organic materials, $0.1 \AA \mathrm{s}^{-1}$ for LiF, and $5 \AA \mathrm{s}^{-1}$ for $\mathrm{Al}$, respectively. Electroluminescence (EL) spectra and luminance intensities were recorded by Photo Research PR655. The current density (J) and driving voltage (V) characteristics were measured by Keithley 2400 simultaneously. External quantum efficiency (EQE) was calculated from the current density, luminance, and EL spectrum, assuming a Lambertian distribution.

Theoretical calculations. The computational models were extracted from the X-ray crystal structures of the representative molecules. In order to eliminate the error of the crystal structure while maintaining the configuration of molecules in the crystal, the ground-state geometries $\left(\mathrm{S}_{0}\right)$ were optimized by applying constraining potentials at carbon and nitrogen atoms using xtb-6.3.3 $3^{26,27}$. Unrestricted DFT method were used to get the lowest-energy triplet states $\left(\mathrm{T}_{1}\right)$ using Gaussian $09 \mathrm{D} 01^{28}$. Vibrational frequency calculations were used to confirm that the optimized structures were minima of corresponding potential energy surfaces, as characterized by all real vibrational frequencies. The excited state properties and Spin-orbit coupling matrix of $\mathrm{T}_{1}$ and $\mathrm{S}_{0}$ were calculated by TD-DFT using ORCA 4.2.1 ${ }^{29}$, All DFT/TD-DFT calculations were carried out at the theory level of M06-2X/def2-SVP 30,31 . 


\section{Data availability}

The X-ray crystallographic data for the structures reported here have been deposited at the Cambridge Crystallographic Data Centre (CCDC) under deposition numbers CCDC 2063597 and 2063598. These data can be obtained free of charge from the Cambridge Crystallographic Data Centre at www.ccdc.cam.ac.uk/data_request/cif. Source data are provided with this paper. 


\section{References}

1. Tang, C. W. \& VanSlyke, S. A. Organic electroluminescent diodes. Appl. Phys. Lett. 51, 913-915 (1987).

2. Baldo, M. A. et al. Highly efficient phosphorescent emission from organic electroluminescent devices. Nature 395, 151154 (1998).

3. Uoyama, H., Goushi, K., Shizu, K., Nomura, H. \& Adachi, C. Highly efficient organic light-emitting diodes from delayed fluorescence. Nature 492, 234-238 (2012).

4. Ai, X. et al. Efficient radical-based light-emitting diodes with doublet emission. Nature 563, 536-540 (2018).

5. Li, W. et al. Employing similar to $100 \%$ Excitons in OLEDs by Utilizing a Fluorescent Molecule with Hybridized Local and Charge-Transfer Excited State. Adv. Funct. Mater. 24, 1609-1614 (2014).

6. Wang, H. et al. Amorphous lonic Polymers with Color-Tunable Ultralong Organic Phosphorescence. Angew. Chem. Int. Ed. 58, 18776-18782 (2019).

7. Thomas, H. et al. Aromatic Phosphonates: A Novel Group of Emitters Showing Blue Ultralong Room Temperature Phosphorescence. Adv. Mater. 32, 2000880 (2020).

8. Liao, Q. Y. et al. 9,9-Dimethylxanthene Derivatives with Room-Temperature Phosphorescence: Substituent Effects and Emissive Properties. Angew. Chem. Int. Ed. 59, 9946-9951 (2020).

9. Zhang, X. et al. Ultralong UV/mechano-excited room temperature phosphorescence from purely organic cluster excitons. Nat. Commun. 10, 5161 (2019).

10. Ma, X., Wang, J. \& Tian, H. Assembling-Induced Emission: An Efficient Approach for Amorphous Metal-Free Organic Emitting Materials with Room Temperature Phosphorescence. Acc. Chem. Res. 52, 738-748 (2019).

11. Ma, H. L., Peng, Q., An, Z. F., Huang, W. \& Shuai, Z. G. Efficient and Long-Lived Room-Temperature Organic Phosphorescence: Theoretical Descriptors for Molecular Designs. J. Am. Chem. Soc. 141, 1010-1015 (2019).

12. Chen, C. et al. Carbazole isomers induce ultralong organic phosphorescence. Nat. Mater. 20, 175-180 (2021).

13. Wang, T. et al. Aggregation-Induced Dual-Phosphorescence from Organic Molecules for Nondoped Light-Emitting Diodes. Adv. Mater. 31, 1904273 (2019).

14. Wang, J. X. et al. Purely Organic Phosphorescence Emitter-Based Efficient Electroluminescence Devices. J. Phys. Chem. Lett. 10, 5983-5988 (2019).

15. Liang, B., Wang, J., Cheng, Z., Wei, J. \& Wang, Y. Exciplex-Based Electroluminescence: Over 21\% External Quantum Efficiency and Approaching 100 Im/W Power Efficiency. J. Phys. Chem. Lett. 10, 2811-2816 (2019).

16. Baldo, M. A., Thompson, M. E. \& Forrest, S. R. High-efficiency fluorescent organic light-emitting devices using a phosphorescent sensitizer. Nature 403, 750-753 (2000).

17. Cheng, G., Zhang, Y., Zhao, Y., Liu, S. \& Ma, Y. Improved efficiency for white organic light-emitting devices based on phosphor sensitized fluorescence. Appl. Phys. Lett. 88, 083512 (2006).

18. Paterson, L. et al. Perspectives of Unicolored Phosphor-Sensitized Fluorescence. Adv. Electron. Mater. 5, 1900646 (2019).

19. Kim, H. G. et al. Triplet Harvesting by a Fluorescent Emitter Using a Phosphorescent Sensitizer for Blue Organic-LightEmitting Diodes. ACS Appl. Mater. Interfaces 11, 26-30 (2019).

20. Lee, K. H. \& Lee, J. Y. Phosphor sensitized thermally activated delayed fluorescence organic light-emitting diodes with ideal deep blue device performances. J. Mater. Chem. C 7, 8562-8568 (2019).

21. Yao, J. W. et al. High efficiency blue/green/yellow/red fluorescent organic light-emitting diodes sensitized by phosphors: general design rules and electroluminescence performance analysis. J. Mater. Chem. C 7, 11293-11302 (2019).

22 Kim, H. G., Kim, K. H. \& Kim, J. J. Highly Efficient, Conventional, Fluorescent Organic Light-Emitting Diodes with Extended Lifetime. Adv. Mater. 29, 1702159 (2017).

23. Heimel, P. et al. Unicolored phosphor-sensitized fluorescence for efficient and stable blue OLEDs. Nat. Commun. 9, 4990 (2018).

24. Burghart, A. et al. Energy transfer cassettes based on BODIPY dyes. Chem. Commun., 2203-2204 (2000). 
25. An, Z. et al. Stabilizing triplet excited states for ultralong organic phosphorescence. Nat. Mater. 14, 685-690 (2015).

26. Bannwarth, C., Ehlert, S. \& Grimme, S. GFN2-xTB-An Accurate and Broadly Parametrized Self-Consistent Tight-Binding Quantum Chemical Method with Multipole Electrostatics and Density-Dependent Dispersion Contributions. J. Chem. Theory Comput. 15, 1652-1671 (2019).

27. Bannwarth, C. et al. Extended tight-binding quantum chemistry methods. WIREs Comput. Mol. Sci. 11, e01493 (2021). 28. Frisch, M. J., Trucks, G. W., Schlegel, H. B., Scuseria, G. E., Robb, M. A., Cheeseman, J. R., Scalmani, G., Barone, V., Petersson, G. A., Nakatsuji, H., Li, X., Caricato, M., Marenich, A. V., Bloino, J., Janesko, B. G., Gomperts, R., Mennucci, B., Hratchian, H. P., Ortiz, J. V., Izmaylov, A. F., Sonnenberg, J. L., Williams, Ding, F., Lipparini, F., Egidi, F., Goings, J., Peng, B., Petrone, A., Henderson, T., Ranasinghe, D., Zakrzewski, V. G., Gao, J., Rega, N., Zheng, G., Liang, W., Hada, M., Ehara, M., Toyota, K., Fukuda, R., Hasegawa, J., Ishida, M., Nakajima, T., Honda, Y., Kitao, O., Nakai, H., Vreven, T., Throssell, K., Montgomery Jr., J. A., Peralta, J. E., Ogliaro, F., Bearpark, M. J., Heyd, J. J., Brothers, E. N., Kudin, K. N., Staroverov, V. N., Keith, T. A., Kobayashi, R., Normand, J., Raghavachari, K., Rendell, A. P., Burant, J. C., Iyengar, S. S., Tomasi, J., Cossi, M., Millam, J. M., Klene, M., Adamo, C., Cammi, R., Ochterski, J. W., Martin, R. L., Morokuma, K., Farkas, O., Foresman, J. B., Fox, D. J. Gaussian 09 Rev. D.01, Gaussian, Inc., Wallingford, CT (2016).

29. Neese, F. Software update: the ORCA program system, version 4.0. WIREs Comput. Mol. Sci. 8, e1327 (2018).

30. Zhao, Y. \& Truhlar, D. G. The M06 suite of density functionals for main group thermochemistry, thermochemical kinetics, noncovalent interactions, excited states, and transition elements: two new functionals and systematic testing of four M06-class functionals and 12 other functionals. Theor. Chem. Acc. 120, 215-241 (2008).

31. Weigend, F. \& Ahlrichs, R. Balanced basis sets of split valence, triple zeta valence and quadruple zeta valence quality for H to Rn: Design and assessment of accuracy. Phys. Chem. Chem. Phys. 7, 3297-3305 (2005). 


\section{Supplementary Information}

General Information: The UV-Vis absorption spectra recorded by a Shimadzu UV-2550 spectrophotometer. The emission spectra were measured using an Edinburgh FLSP920 fluorescence spectrophotometer equipped with a xenon arc lamp (Xe750). Both fluorescence and phosphorescent spectra at low temperature $(77 \mathrm{~K})$ were recorded by Ocean Optics QE Pro with a $365 \mathrm{~nm}$ Ocean Optics LLS excitation source. The absolute fluorescence quantum yields of films were measured on Edinburgh FLS920 steady state fluorimeter utilizing an integrating sphere (excited at $315 \mathrm{~nm}$ ). Transient PL decay, temperature dependent transient PL decay and time-dependent transient PL decay were measured using FLS920 fluorescence lifetime measurement system with $365 \mathrm{~nm}$ LED excitation source and a microsecond flash-lamp (uF750).

Theoretical Calculations method: The computational models were extracted from the X-ray crystal structures of the representative molecules. In order to eliminate the error of the crystal structure while maintaining the configuration of molecules in the crystal, the ground-state geometries $\left(\mathrm{S}_{0}\right)$ were optimized by applying constraining potentials at carbon and nitrogen atoms using xtb-6.3.3. ${ }^{1-2}$ Unrestricted DFT method were used to get the lowest-energy triplet states $\left(\mathrm{T}_{1}\right)$ using Gaussian 09 D01. ${ }^{3}$ Vibrational frequency calculations were used to confirm that the optimized structures were minima of corresponding potential energy surfaces, as characterized by all real vibrational frequencies. The excited state properties and Spin-orbit coupling matrix of $\mathrm{T}_{1}$ and $\mathrm{S}_{0}$ were calculated by TD-DFT using ORCA 4.2.1 ${ }^{4}$, All DFT/TD-DFT calculations were carried out at the theory level of M06-2X/def2-SVP. ${ }^{5-6}$

Single Crystal Structure: The crystal-PE was prepared by slowly evaporating a mixed solution of $\mathrm{CH}_{2} \mathrm{Cl}_{2}$ and petroleum ether (PE), while the crystal-EtOH was generated by delay evaporation of a mixed solution of $\mathrm{CH}_{2} \mathrm{Cl}_{2}$ and ethyl alcohol (EtOH). Diffraction data were collected on a Rigaku RAXIS-PRID diffractometer using the $\omega$-scan mode with graphite monochromator $\mathrm{Mo} \bullet \mathrm{K}_{\alpha}$ radiation. The structure was solved using SHELXT and refined with SHELXL. Non-hydrogen atoms were refined anisotropically. The positions of hydrogen atoms were calculated and refined isotropically. CCDC 2063597 and 2063598 contain the supplementary crystallographic data for this paper, and the data can be obtained free of charge from The Cambridge Crystallographic Data Centre via www.ccdc.cam.ac.uk/data_request/cif.

Device Fabrication and Characterization: Glass substrates pre-coated with indium tin oxide (ITO) 
with a sheet resistance of $15 \Omega$ per square were thoroughly cleaned in ultrasonic bath of tetrahydrofuran, detergent, deionized water, acetone and isopropyl alcohol and treated with plasma for 5 min in sequence. Organic layers were deposited onto the ITO-coated glass substrates by thermal evaporation under high vacuum $\left(<5 \times 10^{-4} \mathrm{~Pa}\right)$. Cathode was patterned using a shadow mask with an array of $2.0 \mathrm{~mm} \times 2.5 \mathrm{~mm}$ openings. Deposition rates are $1 \AA \mathrm{s}^{-1}$ for organic materials, $0.1 \AA \mathrm{s}^{-1}$ for LiF, and $5 \AA \mathrm{s}^{-1}$ for $\mathrm{Al}$, respectively. Electroluminescence (EL) spectra and luminance intensities were recorded by Photo Research PR655. The current density (J) and driving voltage (V) characteristics were measured by Keithley 2400 simultaneously. External quantum efficiency (EQE) was calculated from the current density, luminance, and EL spectrum, assuming a Lambertian distribution.

\section{Estimation of the Förster radius:}

The Förster radius $\left(\mathrm{R}_{0}\right)$ was estimated from the overlap $J(\lambda)$ of extinction spectrum of rubrene and PL spectrum of $\beta$-DPTZN by using the following equation:

$$
R_{0}^{6}=\frac{9000(\ln 10) \kappa^{2} \Phi_{D}}{128 \pi^{5} N_{A} n^{4}} J(\lambda)
$$

Where $\kappa^{2}$ is orientation factor, $\Phi_{D}$ is the PL quantum yield of donor, $N_{A}$ is Avogadro's number, $\mathrm{n}$ is the refractive index of the solvent. $J(\lambda)$ is calculated from the following equation:

$$
J(\lambda)=\frac{\int_{0}^{\infty} F_{D}(\lambda) \varepsilon_{A}(\lambda) \lambda^{4} d \lambda}{\int_{0}^{\infty} F_{D}(\lambda) d \lambda}
$$

We used $\Phi_{D}=0.38, \kappa^{2}=0.667, \mathrm{n}=1.7$ and $J(\lambda)=7.8 \times 10^{14} \mathrm{M}^{-1} \mathrm{~cm}^{-1} \mathrm{~nm}^{4} . R_{0}$ is calculated to be $29.64 \mathrm{~nm}$. 

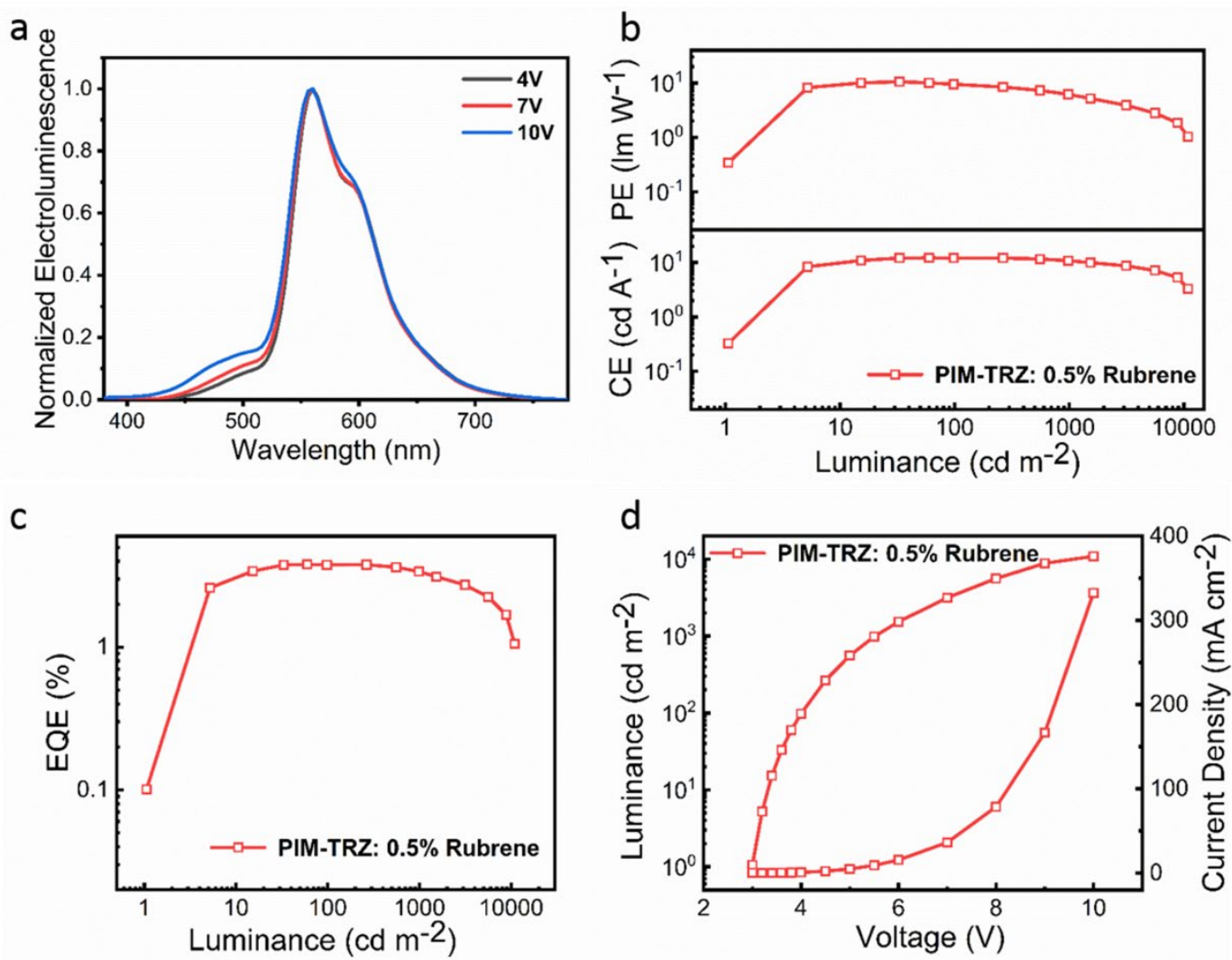

Fig. S1. (a) EL spectra of device 6. (b) PE-Brightness-CE characteristics of device 6. (c) EQEBrightness and (d) Current density-Voltage-Brightness (J-V-L) characteristics of device 6. 

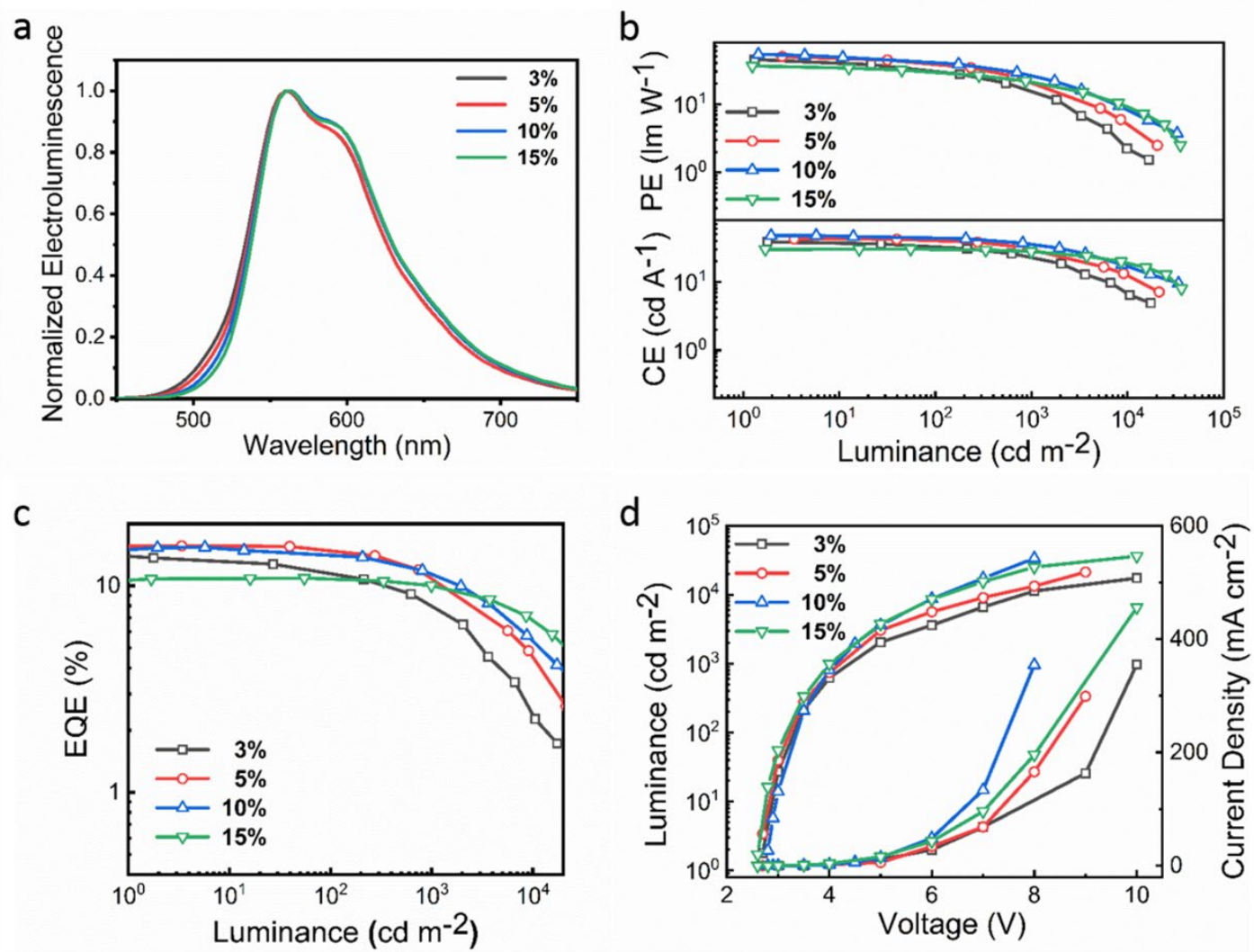

Fig. S2. (a) EL spectra of device 7-10 at $100 \mathrm{~cd} \mathrm{~m}^{-2}$. (b) PE-Brightness-CE characteristics of devices 7-10. (c) EQE-Brightness and (d) Current density-Voltage-Brightness (J-V-L) characteristics of devices 7-10. (Y represent the doping concentration of $\beta$-DPTZN. device 7, Y=3\%; device $8, Y=5 \%$; device $9, \mathrm{Y}=10 \%$; device $10, \mathrm{Y}=15 \%$ )
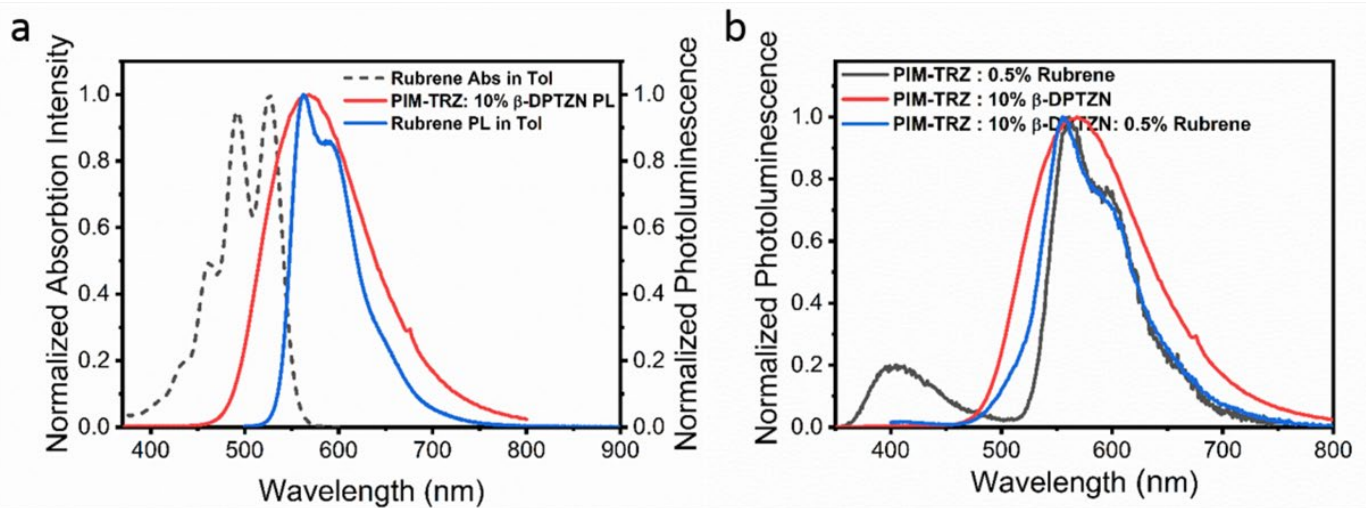

Fig. S3. (a) The absorbtion spectrum of rubrene in toluene solution and PL spectra of [PIM-TRZ:10 wt $\%$ $\beta$-DPTZN] blending film and rubrene in toluene solution. (b) PL spectra of [PIM-TRZ:0.5 wt $\%$ rubrene] blending film, [PIM-TRZ:10 wt $\% \beta$-DPTZN] blending film and [PIM-TRZ:10 wt $\% \beta$-DPTZN:0.5 wt $\%$ rubrene] blending film. 

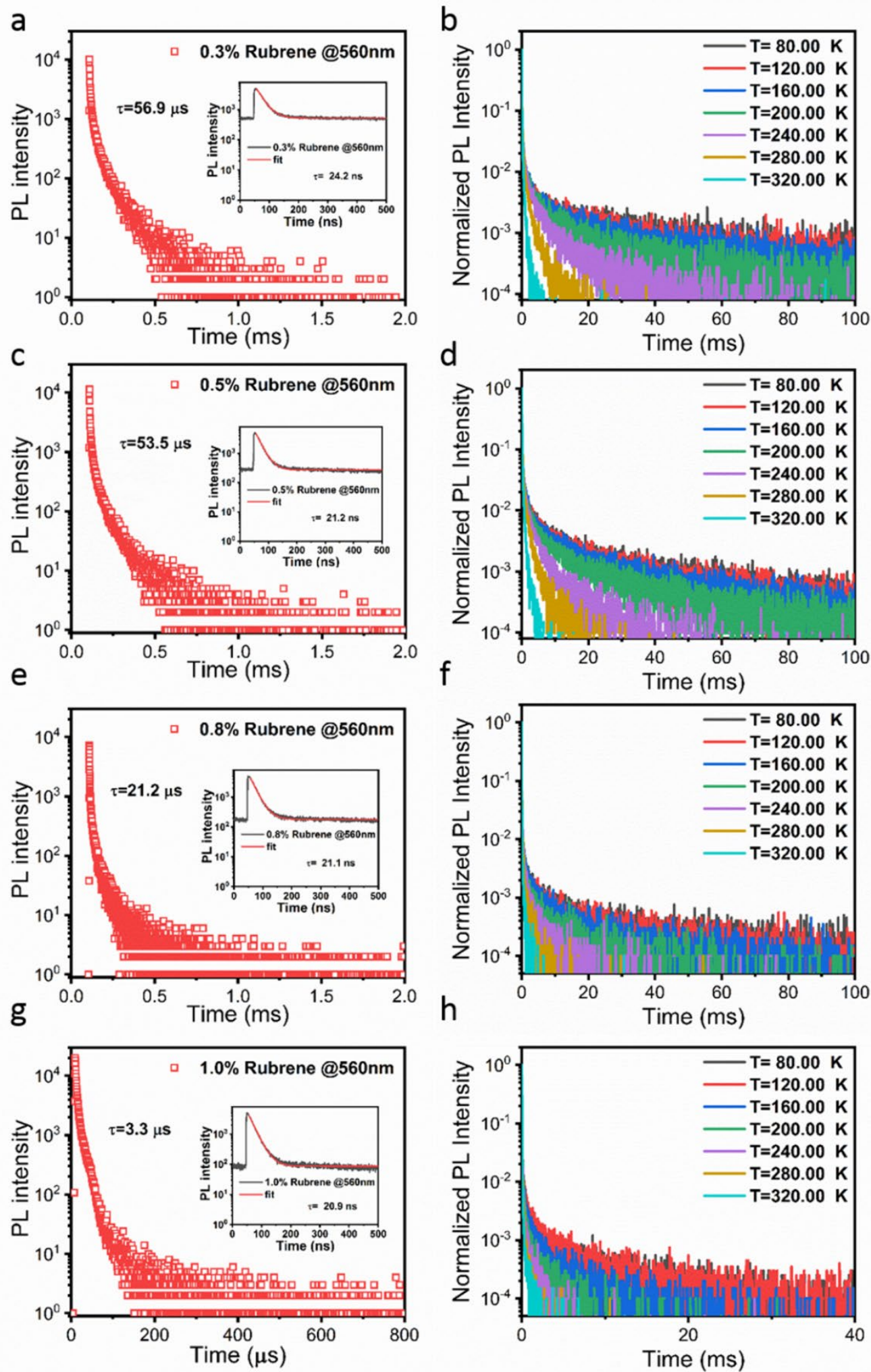

Fig. S4. Transient PL spectra of [PIM-TRZ:10 wt $\% \beta$-DPTZN:X wt $\%$ rubrene] blending film (left) and temperature-dependent transient PL spectra of [PIM-TRZ:10 wt $\% \beta$-DPTZN:X wt $\%$ rubrene] blending film (right) $(\mathrm{X}=0.3,0.5,0.8,1.0)$. 
a

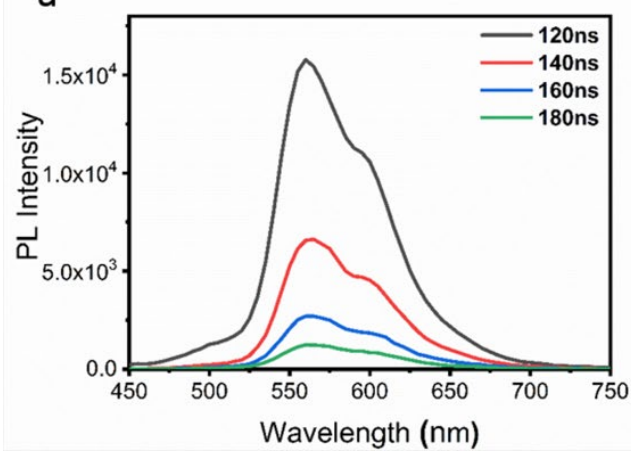

b

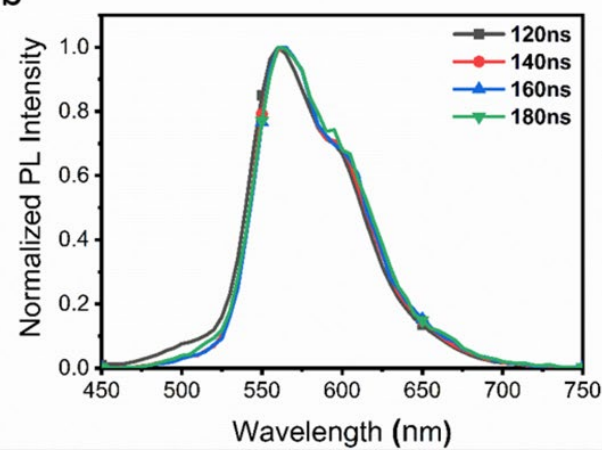

Fig. S5. Time-dependent PL spectra of [PIM-TRZ:10 wt $\% \beta$-DPTZN:0.3 wt $\%$ rubrene].
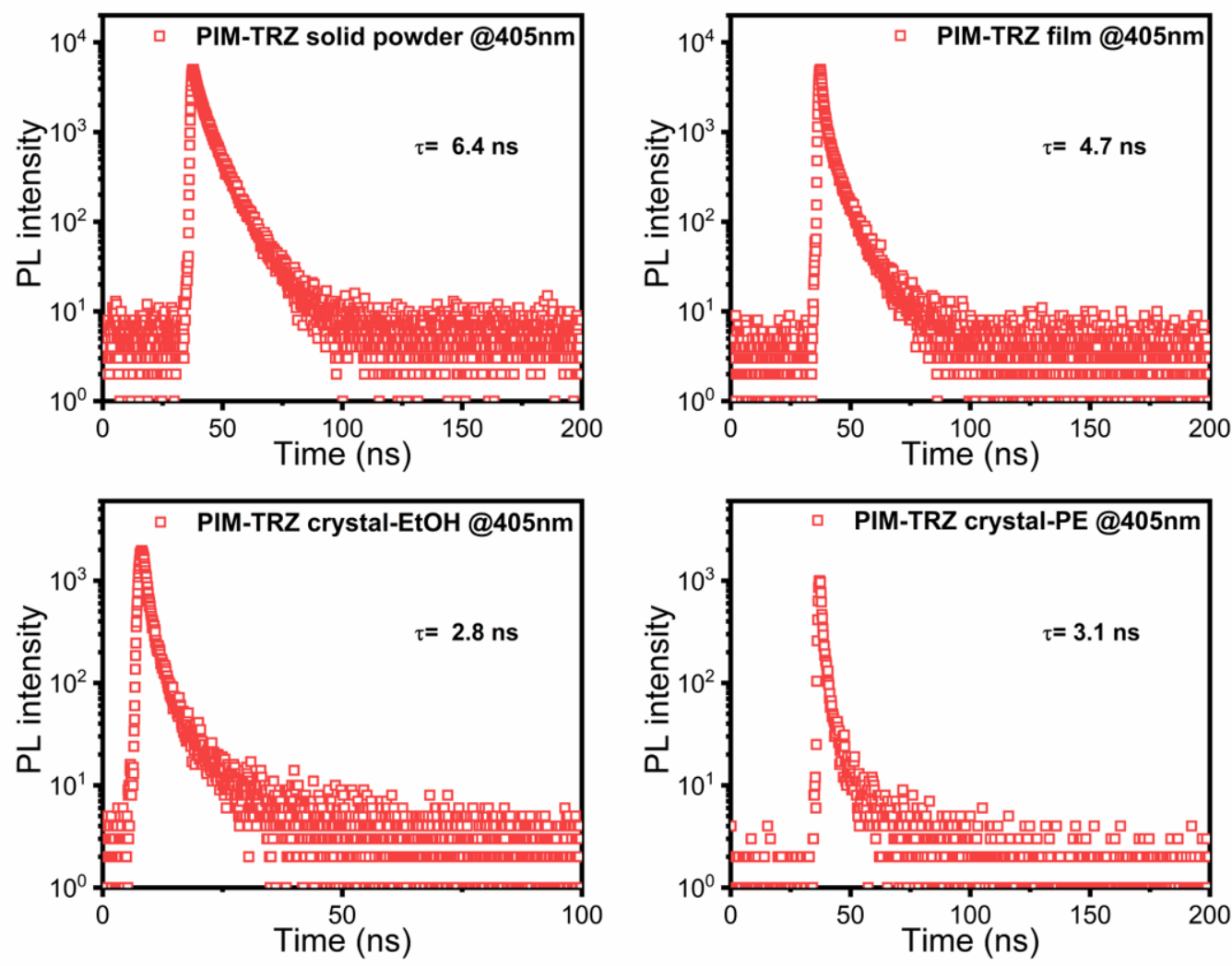

Fig. S6. Transient PL spectra of PIM-TRZ in different states of solid powder, film, crystal EtOH and crystal-PE recorded at $405 \mathrm{~nm}$. 

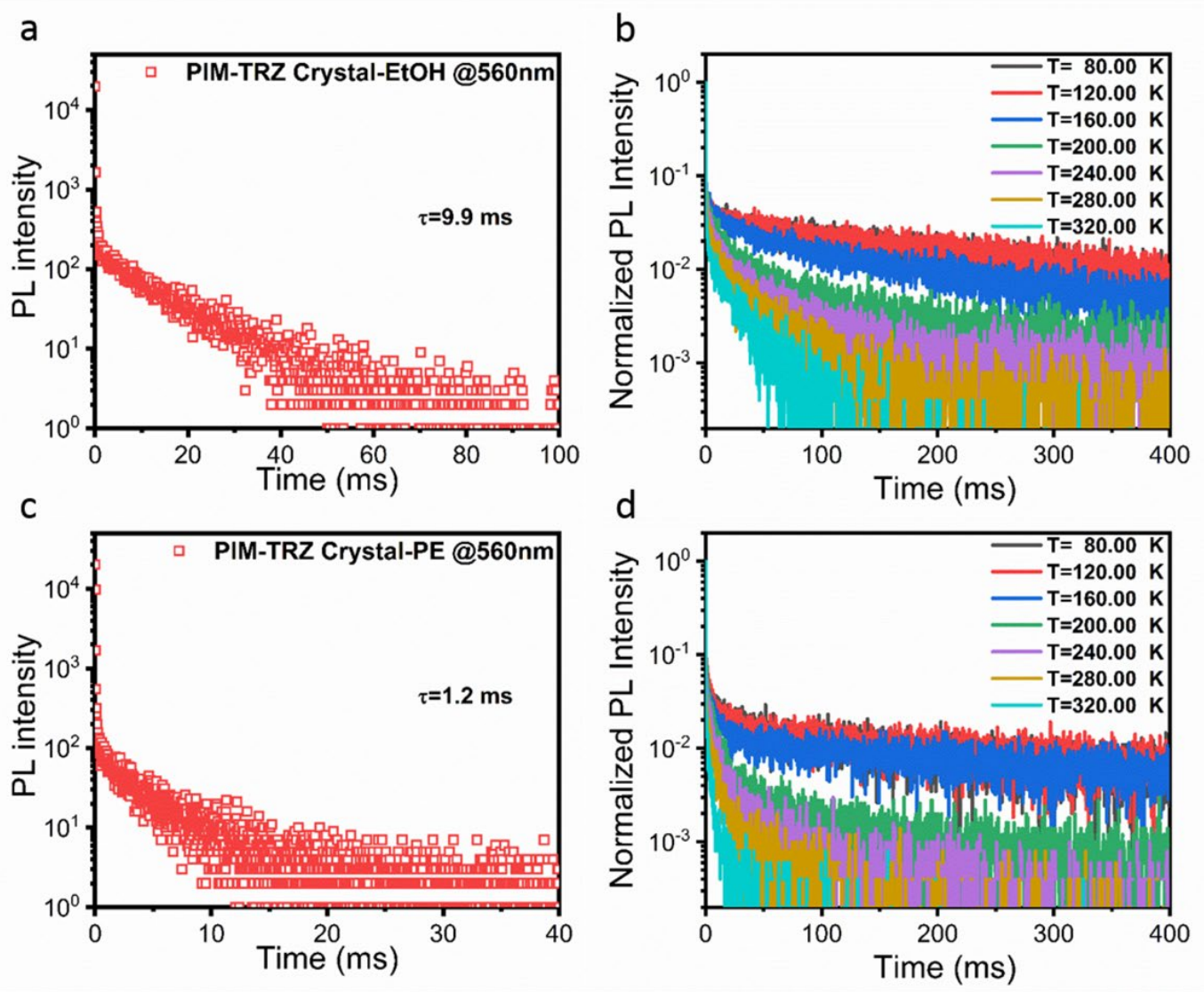

Fig. S7. (a) Transient PL spectra of PIM-TRZ crystal-EtOH. (b) Temperature-dependent transient PL spectra of PIM-TRZ crystal-EtOH. (c) Transient PL spectra of PIM-TRZ crystal-PE. d) Temperaturedependent transient PL spectra of PIM-TRZ crystal-PE. 

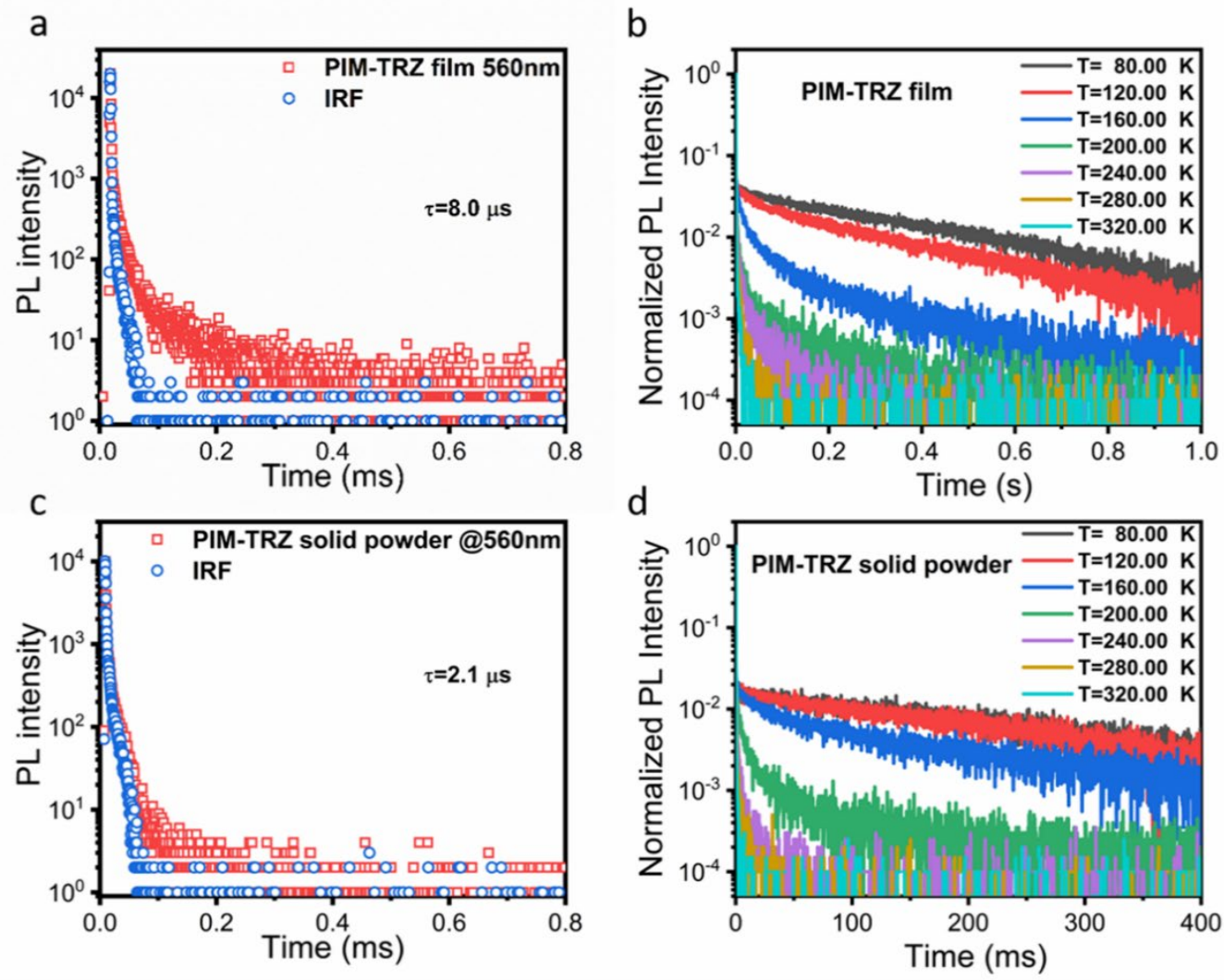

Fig. S8. (a) Transient PL spectra of PIM-TRZ film. (b) Temperature-dependent transient PL spectra of PIM-TRZ film. (c) Transient PL spectra of PIM-TRZ solid powder. (d) Temperature-dependent transient PL spectra of PIM-TRZ solid powder. 


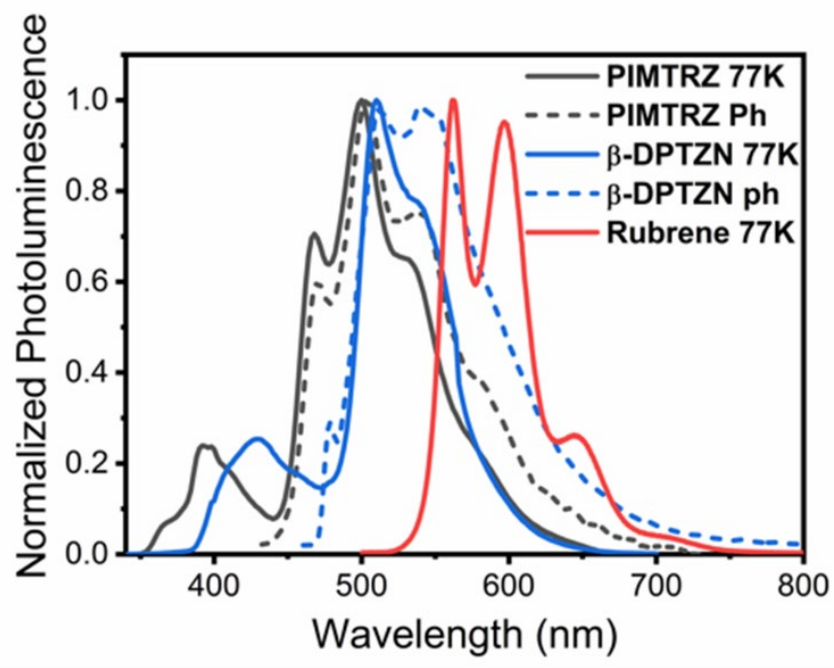

Fig. S9. The PL spectra of PIM-TRZ, $\beta$-DPTZN and rubrene in toluene solution at $77 \mathrm{~K}$ and phosphorescence spectra of PIM-TRZ and $\beta$-DPTZN in toluene solution at $77 \mathrm{~K}$.

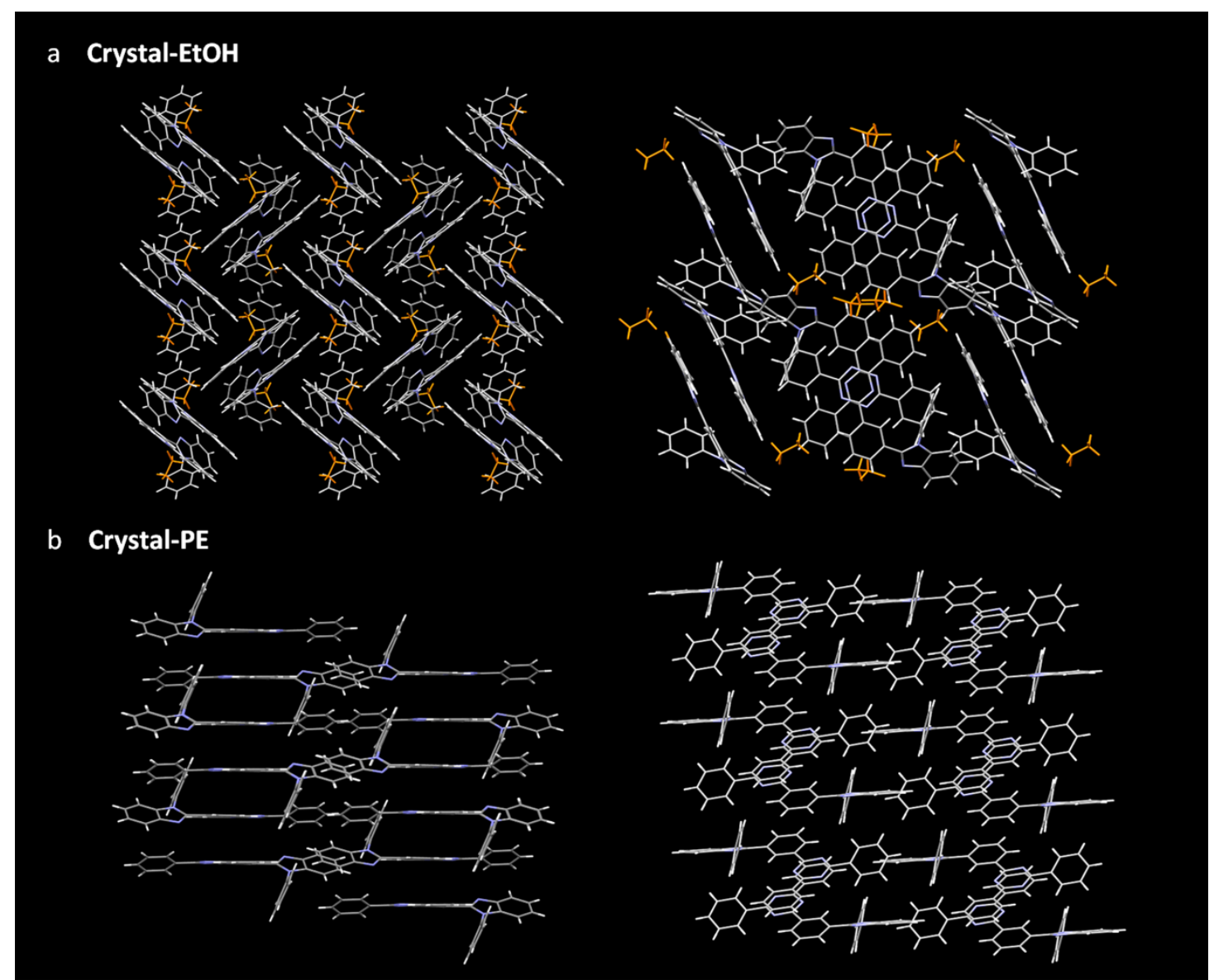

Figure S10. The molecular packing modes of crystal-EtOH and crystal-PE. 
Table S1. PLQY and radiative decay times listed with increasing acceptor concentration.

\begin{tabular}{ccccc}
\hline $\begin{array}{c}\text { Fluorescence Emitter } \\
\text { Concentration } \\
{[\%]}\end{array}$ & 0.3 & 0.5 & 0.8 & 1.0 \\
\hline $\mathbf{P L Q Y}[\%]$ & 60.3 & 56.5 & 56.3 & 55.0 \\
$\boldsymbol{\tau}_{\mathrm{rad}[\mu \mathrm{s}]}$ & 56.9 & 53.5 & 21.2 & 3.3 \\
\hline
\end{tabular}

Table S2. Electroluminescent parameters of the devices 7-10.

\begin{tabular}{ccccccc}
\hline Device & $\begin{array}{c}\mathrm{V}_{\text {on }} \\
{[\mathrm{V}]^{[\mathrm{a}]}}\end{array}$ & $\begin{array}{c}\mathrm{L}_{\max } \\
{\left[\mathrm{cd} \mathrm{m}^{-2}\right]^{[\mathrm{b}]}}\end{array}$ & $\begin{array}{c}\mathrm{CE} \\
{\left[\mathrm{cd} \mathrm{A}^{-1}\right]^{[\mathrm{c}]}}\end{array}$ & $\begin{array}{c}\text { PE } \\
{[\mathrm{lm} \mathrm{W}]^{[\mathrm{c}]}}\end{array}$ & $\begin{array}{c}\text { EQE } \\
{[\%]^{[\mathrm{c}]}}\end{array}$ & $\begin{array}{c}\text { CIE } \\
(x, y)^{[\mathrm{d}]}\end{array}$ \\
\hline Device 7 & 2.7 & 17410 & $38.8,30.6,25.9$ & $45.1,27.4,20.4$ & $13.6,10.8,9.1$ & $0.48,0.51$ \\
Device 8 & 2.7 & 21310 & $42.6,38.2,32.6$ & $49.5,34.3,25.6$ & $15.6,14.0,11.9$ & $0.48,0.51$ \\
Device 9 & 2.8 & 33820 & $47.8,42.9,37.0$ & $53.6,38.5,29.1$ & $15.3,13.8,11.9$ & $0.47,0.52$ \\
Device 10 & 2.6 & 36400 & $30.2,29.3,27.8$ & $36.2,26.3,21.8$ & $10.9,10.5,10.0$ & $0.49,0.50$ \\
\hline
\end{tabular}

${ }^{[a]}$ turn-on votage at $1.0 \mathrm{~cd} \mathrm{~m}^{-2} .{ }^{[b]}$ maximum luminance. ${ }^{[c]}$ in the order of maximum, then the values recorded at 100 and $1000 \mathrm{~cd} \mathrm{~m}^{-2}$. ${ }^{[\mathrm{d}]}$ recorded at $100 \mathrm{~cd} \mathrm{~m}^{-2}$.

Table S3. Calculate SOCME of PIM-TRZ crystal-PE and crystal-EtOH.

CALCULATE SOCME $\left(\mathrm{cm}^{-1}\right)$

\begin{tabular}{ccc} 
Conformation & $<\mathrm{T}_{1}\left|\mathrm{H}_{\mathrm{SO}}\right| \mathrm{S}_{1}>$ & $<\mathrm{T}_{1}\left|\mathrm{H}_{\mathrm{SO}}\right| \mathrm{S}_{0}>$ \\
Crystal-PE & 0.790 & 0.785 \\
Crystal-EtOH & 0.371 & 0.703 \\
\hline
\end{tabular}

\section{References}

1. Bannwarth, C., Ehlert, S. \& Grimme, S. GFN2-xTB-An Accurate and Broadly Parametrized SelfConsistent Tight-Binding Quantum Chemical Method with Multipole Electrostatics and DensityDependent Dispersion Contributions. J. Chem. Theory Comput. 15, 1652-1671 (2019).

2. Bannwarth, C. et al. Extended tight-binding quantum chemistry methods. WIREs Comput. Mol. Sci. 11, e01493 (2021). 
3. Frisch, M. J., Trucks, G. W., Schlegel, H. B., Scuseria, G. E., Robb, M. A., Cheeseman, J. R., Scalmani, G., Barone, V., Petersson, G. A., Nakatsuji, H., Li, X., Caricato, M., Marenich, A. V., Bloino, J., Janesko, B. G., Gomperts, R., Mennucci, B., Hratchian, H. P., Ortiz, J. V., Izmaylov, A. F., Sonnenberg, J. L., Williams, Ding, F., Lipparini, F., Egidi, F., Goings, J., Peng, B., Petrone, A., Henderson, T., Ranasinghe, D., Zakrzewski, V. G., Gao, J., Rega, N., Zheng, G., Liang, W., Hada, M., Ehara, M., Toyota, K., Fukuda, R., Hasegawa, J., Ishida, M., Nakajima, T., Honda, Y., Kitao, O., Nakai, H., Vreven, T., Throssell, K., Montgomery Jr., J. A., Peralta, J. E., Ogliaro, F., Bearpark, M. J., Heyd, J. J., Brothers, E. N., Kudin, K. N., Staroverov, V. N., Keith, T. A., Kobayashi, R., Normand, J., Raghavachari, K., Rendell, A. P., Burant, J. C., Iyengar, S. S., Tomasi, J., Cossi, M., Millam, J. M., Klene, M., Adamo, C., Cammi, R., Ochterski, J. W., Martin, R. L., Morokuma, K., Farkas, O., Foresman, J. B., Fox, D. J. Gaussian 09 Rev. D.01, Gaussian, Inc., Wallingford, CT (2016).

4. Neese, F. Software update: the ORCA program system, version 4.0. WIREs Comput. Mol. Sci. 8, e1327 (2018).

5. Zhao, Y. \& Truhlar, D. G. The M06 suite of density functionals for main group thermochemistry, thermochemical kinetics, noncovalent interactions, excited states, and transition elements: two new functionals and systematic testing of four M06-class functionals and 12 other functionals. Theor. Chem. Acc. 120, 215-241 (2008).

6. Weigend, F. \& Ahlrichs, R. Balanced basis sets of split valence, triple zeta valence and quadruple zeta valence quality for $\mathrm{H}$ to Rn: Design and assessment of accuracy. Phys. Chem. Chem. Phys. 7, 3297-3305 (2005). 